\title{
Análisis de la seguridad laboral mediante la medición de los niveles de estrés laboral
}

\author{
Omar Alejandro Pérez Cruz / omar_perez@ucol.mx \\ Universidad de Colima
}

Recibido: 29 de febrero de 2012

Aceptado: 7 de septiembre de 2012

\section{RESUMEN:}

La interacción diaria con distintas personas, los cambios de horarios y el estrés que causa atender emergencias de todo tipo genera en el personal un desgaste no solo físico, sino también emocional y psicológico que influye en su desempeño laboral. Este estudio examinó los niveles de estrés laboral en el Sistema de Emergencias del estado de Colima, México, utilizando el modelo de Maslach Burnout Inventory (MBI). Los resultados indican que la mayoría de estos ejecutivos están a riesgo de desarrollar el síndrome de burnout. Esto puede llevar a diseñar estrategias que intervengan en el manejo del estrés laboral y que se orienten a desarrollar el sentido de pertenencia del grupo de trabajo.

Palabras clave: burnout, estrés, seguridad, emergencias

\section{ABSTRACT:}

Daily interaction with different people, schedule changes and the stress of dealing with emergencies of all types generated in the staff wear not only physical, but also emotional and psychological impact on their job performance. This study examined the levels of stress in the Emergency State of Colima, Mexico, using the model of Maslach Burnout Inventory (MBI).The results indicate that most of these executives are at risk for burnout. This can lead to design strategies involved in managing work stress and that aim to develop a sense of ownership of the working group.

Keywords: burnout, stress, security, emergency 


\section{INTRODUCCIÓN}

Bernaldo de Quirós-Aragón y Labrador-Encinas (2007), Guerrero (2003), Fernández (2010), Peralta Gómez (2006) y Benevides Pereira, Justo, Batista Gomes, Martins Silva y Volpato (2003), entre otros, han centrado sus estudios en las manifestaciones del estrés y su relación con diferentes aspectos del ámbito laboral. Estas son situaciones que afectan la calidad de vida y las distintas áreas de funcionamiento del trabajador como son lo social, lo familiar, lo académico y lo laboral. De esta manera, el estrés laboral genera altos costos laborales y sociales, lo que ha llevado a que diversas organizaciones como la Unión Europea, la Organización Internacional del Trabajo y la Organización Mundial de la Salud, insistan de manera cada vez más recurrente sobre la importancia de su detección y control.

De acuerdo con Cano (2012) el término estrés proviene de la física y de la arquitectura, y hace referencia a la fuerza que se aplica a un objeto y que puede deformarlo o romperlo. En la gestión de recursos humanos, el estrés suele hacer referencia a ciertos acontecimientos que suscitan fuertes demandas de energía mental y física en el individuo, que lo pueden llevar al extremo de agotar su capacidad de afrontamiento.

A partir de 1974, año en que Freudenberg escribió por primera vez acerca de las experiencias de desgaste profesional, las investigaciones sobre el tema se han multiplicado (Schaufeli, Leiter y Maslach, 2008). Hoy día, luego de 35 años de que se acuñó este término, se han producido más de 3,000 investigaciones, artículos y libros relacionados con el burnout (BO en adelante), lo que demuestra el creciente interés de los científicos en el tema.

El BO es un síndrome que puede afectar una gran diversidad de profesiones muy diferentes, desde sanitarias, docentes, hasta policiales. Debido a que la mayoría de los puestos de trabajo son cada vez más exigentes, el $\mathrm{BO}$ se manifiesta no solo en profesiones asistenciales sino que se extiende a otros grupos profesionales que incluye desde administradores hasta personal no cualificado.

Por lo general, este síndrome suele presentarse preferentemente en aquellas ocupaciones que tienen que atender e interactuar con frecuencia de forma asistencial con algún tipo de usuario o "cliente" 
que presenta necesidades más o menos importantes e imperiosas. El modelo operativo inicial fue desarrollado por Maslach y Jackson (1986), quienes definieron el BO como un síndrome caracterizado por cansancio emocional (EE), Despersonalización (D) y Realización personal (RP). Las personas afectadas por el síndrome desarrollan síntomas muy variados que interfieren negativamente en su trabajo, en su salud y en su vida personal. A estas tres dimensiones se añaden la tensión y la frustración por no obtener los resultados esperados en su trabajo.

Moreno, Oliver, Pastor y Aragoneses (1990) definen el BO como un proceso de adaptación al estrés laboral, que se caracteriza por desorientación profesional, desgaste, sentimientos de culpa por falta de éxito profesional, frialdad o distanciamiento emocional y aislamiento.

Las diferentes definiciones del $\mathrm{BO}$ comparten algunas características. En todas ellas predominan más los síntomas mentales que los físicos. Asimismo, todas destacan el cansancio mental o emocional, la fatiga y la depresión, siendo el agotamiento emocional el más característico de todos (Bernaldo de Quirós-Aragón y LabradorEncinas (2007); Guerrero (2003); Fernández (2010); Peralta Gómez (2006); Benevides Pereira, Justo, Batista Gomes, Martins Silva y Volpato (2003). En resumen, el BO se clasifica como un síndrome clínico-laboral, que se desencadena por una inadecuada adaptación al trabajo y que conlleva una disminución del rendimiento en el trabajo acompañada por una sensación de baja autoestima.

\section{Problema de investigación}

Desde la perspectiva organizacional se cuestiona de qué manera se afecta el desempeño de los ejecutivos del Centro de Atención a Emergencias 066 del Gobierno del Estado de Colima. Aunque el trabajo que realizan estos ejecutivos no es de manera personal, la atención se da de manera directa, por medio de una llamada telefónica en la que se ofrece asesoría a quién presenta una emergencia.

De esta manera, el interactuar con distintas personas todos los días, sumado al cambio de los turnos de trabajo, tiene un impacto en su rendimiento emocional. Por lo cual, se buscó determinar el 
nivel de $\mathrm{BO}$ en el que se encontraban los ejecutivos del Centro de Atención a Emergencias 066 del Gobierno del Estado de Colima.

\section{Pregunta de inVestigación}

¿Existe el BO en el ejecutivo del centro de atención a emergencias 066 del Gobierno del Estado de Colima?

\section{Objetivo General}

Identificar la incidencia de $\mathrm{BO}$ en el ejecutivo del centro de atención a emergencias 066 del Gobierno del Estado de Colima.

\section{JUSTIFICACIÓN}

Resulta importante determinar si existe $\mathrm{BO}$ en una organización, ya que si se conoce el nivel de incidencia, se pueden establecer determinadas estrategias que ayuden a contrarrestar el índice de $\mathrm{BO}$.

Debido a que los ejecutivos de emergencia del sistema 066 del estado de Colima realizan una función de atención telefónica, es decir, interactúan con los usuarios del sistema, son candidatos para estudiar la incidencia del BO.

\section{El estrés laboral}

De acuerdo con Peiró (1999), a lo largo del siglo XX el estrés se ha convertido en un fenómeno relevante y reconocido socialmente, siendo vinculado con frecuencia al trabajo y a la actividad laboral. De acuerdo con la Agencia Europea para la seguridad y salud en el trabajo (FACTS) al presente, más de una cuarta parte de los trabajadores de la Unión Europea padece estrés laboral (2004).

De acuerdo con Peiró (2005) el estrés laboral surge cuando las demandas del trabajo son altas, y al mismo tiempo, la capacidad de control de las mismas es baja al modelo esfuerzo-recompensa. De esta manera se explica el estrés laboral cuando existe un desequilibrio entre el alto esfuerzo (demandas, obligaciones, etc.) y la baja recompensa (sueldo, estima, etc.).

56 ISSN 1541-8561 FORUM EMPRESARIAL VOL. 17. NÚM. - INVIERNO 2012-2013 
Otros autores, como Dennis (2004) y Guillén (2000) han ampliado el concepto y consideran el estrés como "la condición mental o física que ocurre en cualquier momento en que debemos ajustarnos o adaptarnos al ambiente". Además, se tiende a resaltar la condición de amenaza al individuo como elemento fundamental para la existencia de estrés y la relación cognitiva, emocional y conductual del fenómeno. Por consiguiente, se puede decir que el estrés es un concepto de múltiples aristas, entendido como: (1) una reacción o respuesta del individuo (cambios fisiológicos, reacciones emocionales, cambios conductuales, entre otros) y (2) un estímulo (capaz de provocar una reacción de estrés) o como una interacción entre las características del estímulo y los recursos de afrontamiento del individuo (Cano, 2012).

El estrés conlleva también respuestas fisiológicas ante un estresor ambiental, que puede ser un evento externo u otra situación potencialmente dañina. Lazarus y Folkman (1991) sostienen que las características que hacen que un evento se convierta en un estresor potencial son la incertidumbre, la falta de control sobre la situación, la falta de información, de habilidades o competencias para hacerle frente a la misma, y la modificación de condiciones de comodidad a nuevos escenarios desconocidos o ambiguos.

Lazarus (1984) afirma que entre las emociones que se presentan en este ambiente de trabajo, encontramos rabia, indefensión, decepción, miedo, culpa, fracaso, tristeza, vergüenza, aislamiento, falta de sensibilidad y desánimo.

El estrés laboral tiene varias peculiaridades. Entre ellas se destacan la gran variedad de condiciones organizacionales y ambientales que son capaces de producirlo, la diversidad de respuestas ante condiciones similares, la dificultad de predecir la intensidad y el grado de estrés en un individuo, y los cambios conductuales que genera el estrés prolongado, tales como ausentismo, enfermedad crónica, disminución de la productividad y demandas legales a la empresa.

Gil-Monte y Peiro (1999) proponen que los estresores laborales en general pueden clasificarse en seis categorías: cultura organizacional, estructura organizacional, territorio, tecnología, liderazgo y acoso psicológico. 
$\mathrm{Al}$ respecto, Davis (2004) expone que la cultura organizacional se refiere a la interacción de las personas, la estructura, las políticas y metas que generan una atmósfera o clima. En otras palabras es el "sentir", la "personalidad" o el "carácter" de una organización que puede conducir a un estilo relajado de trabajo o a uno tenso y orientado a las crisis. La cultura determina el ambiente en función de la definición de las conductas apropiadas o inapropiadas de sus miembros, así como los valores compartidos y las sanciones (formales o informales) instituidas para atender las amenazas a lo organizacionalmente aceptado. Las brechas entre lo declarado y lo presentado en realidad son la principal fuente de estrés relacionado con esta condición.

Las acciones, el estilo y los procedimientos de un líder pueden operar como estresores en algunas personas y afectar la producción y la satisfacción laboral. En este sentido, el liderazgo no es necesariamente dictado por una estructura jerárquica dentro de la organización. Davis (2004) argumenta que los estilos de liderazgo que muestran una alta orientación a las tareas y a los resultados, pero que valoran poco a las personas y sus contribuciones, suelen generar estados de apatía, falta de compromiso y desinterés por parte de los trabajadores así como el sentimiento de falta de apreciación por sus contribuciones y, a su vez, desgano por sus actividades laborales cotidianas. Por otro lado, Robbins (2003) sostiene que los estilos de liderazgo participativos en los que el empleado tiene la oportunidad de involucrarse y proponer ideas y acciones que son tomadas en cuenta resultan motivantes para lograr niveles superiores de desempeño.

Por su parte, Disegni (2002) dice que se debe considerar la combinación de factores ambientales y laborales como detonante del fenómeno del estrés, como lo que ocurre con las madres trabajadoras y la doble jornada y doble rol que tienen en la sociedad al también tener un papel familiar de gran trascendencia, situación que es reconocida como contexto potencial de altos índices de estrés.

Entre los efectos conductuales más comunes causados por el estrés se encuentra la propensión a sufrir accidentes. La Organización Internacional del Trabajo (2003) considera el estrés como el factor psicológico principal en la generación de accidentes y enfermedades laborales, condiciones que se elevan a 270 millones de acciden- 
tes por año, 160 millones de enfermedades no mortales y 2 millones de muertes en el mundo.

Según Disegni (2002), el estrés incrementa las conductas de adicción en el abuso de alcohol, tabaco y otras drogas, así como en el abuso en la ingesta de alimentos, lo que fomenta el desarrollo de obesidad, hipertensión y diabetes. De acuerdo con Salgado (2007) el estrés también se relaciona con la pérdida de apetito, la compulsión por comer y con una conducta orientada a sufrir trastornos alimenticios como la anorexia o la bulimia.

Escobar y Gómez (2006) afirman que las afecciones en el aprendizaje y en la memoria derivadas del estrés laboral y psicosocial son señales de alarma importantes pues tienen una repercusión directa en el desempeño de los trabajadores. Estos mismo autores señalan que las mismas se desarrollan principalmente por un exceso en la información que deben manejar los individuos, lo que unido a ambientes que no propician una concentración adecuada impiden que los trabajadores retengan información crítica.

\section{Desarrollo del concepto BO}

El mundo laboral ha experimentado una transformación importante en las últimas décadas. Las nuevas exigencias del trabajo y el desajuste entre los requisitos de los puestos de trabajo en las organizaciones y las posibilidades de rendimiento de cada sujeto han originado la aparición de nuevos riesgos denominados psicosociales, entre ellos el $\mathrm{BO}$, cuya prevalencia se ha ido incrementando y que ha venido a constituirse en un problema social y de salud pública que conlleva un gran costo económico y social, por lo que cualquier esfuerzo para su estudio debe ser bienvenido (Gil-Monte, 2005).

En el contexto de internacionalización económica y la innovación tecnológica, el mundo laboral de la sociedad post-moderna está generando un proceso de grandes cambios pues, mientras que las demandas físicas se han ido reduciendo, las demandas sobre la interacción psicosocial se han ido incrementando. Estas transformaciones experimentadas en el trabajo, en ocasiones, representan mejoras y avances pero, en otras, son causantes de la aparición de una serie de condiciones que conducen a riesgos que afectan el bienestar de la persona. 
Existen diversos estudios auspiciados por la Organización Internacional del Trabajo en países como Alemania, Estados Unidos y Finlandia que muestran la relación entre el incremento de los costos de salud y laborales y la presencia de estrés laboral y BO en sus organizaciones. En Alemania, por ejemplo, el $7 \%$ de las jubilaciones anticipadas se deben a trastornos emocionales derivados del estrés, mientras que el ausentismo relacionado con este fenómeno tiene un costo que supera los 2500 millones de euros (Phyllis, 2000). Aunque en nuestro país, México, no se cuentan con estadísticas precisas sobre la incidencia o los costos de este fenómeno, se conoce que la tercera causa de consultas en el área de Salud Mental del Hospital General de México es la reacción al estrés agudo y grave, y que los costos de salud y la incidencia de consecuencias conductuales y psicológicas asociadas es cada vez mayor (Salgado, 2007).

\section{Metodología}

\section{Diseño de LA INVESTIGACIÓN}

El presente estudio de caso es de carácter descriptivo. De acuerdo con Hernández, Fernández y Baptista (2000), los estudios descriptivos se centran en medir con la mayor precisión posible diversos aspectos, dimensiones o componentes del fenómeno a investigar. De corte no experimental, este tipo de investigación "se realiza sin manipular deliberadamente las variables y se observan los fenómenos tal y como se dan en su contexto natural, para después analizarlos" (184).

Los resultados obtenidos del presente estudio podrán ser utilizados por aquellas organizaciones interesadas en este tema, como guía para desarrollar estrategias de calidad de vida laboral que satisfagan las necesidades de los trabajadores.

\section{UNIDAD DE ANÁLISIS}

Se analizaron las tres dimensiones del síndrome de BO-agotamiento emocional (EE), despersonalización (D) y realización perso- 
nal (RP)-en los ejecutivos del centro de atención a emergencias 066 del Gobierno del Estado de Colima.

\section{TIPO DE MUESTRA}

La muestra fue probabilística. Esta característica de la muestra permitió obtener una representación de la población en general y darle validez a los resultados obtenidos. Así, se eligió una muestra representativa, aleatoria y confiable. Para tal efecto, se utilizó el software SPSS.

\section{TAMAÑO DE LA MUESTRA}

El universo estuvo compuesto por 40 ejecutivos del sistema de emergencias del estado de Colima. El valor que toma al inicio del programa para el error alfa, fue de $10 \%(0.10)$ con un nivel de confianza de $90 \%$ (0.90) lo que equivale a un valor de z de 1.6448, con un nivel de heterogeneidad de 50\%. Por lo tanto, el tamaño de la muestra fue de 26 aplicaciones.

\section{RECOLECCIÓN DE LA INFORMACIÓN}

Se distribuyeron un total de 29 cuestionarios y se recogieron todos, con lo que el porcentaje de respuesta alcanzado fue $100 \%$.

Se realizaron dos sesiones para la aplicación del instrumento, en las cuales se intervino con 4 equipos en total, formando así la muestra de 29 individuos. Para efectos de este estudio, no se consideraron características como el sexo o la edad de los participantes. Los instrumentos fueron aplicados y recogidos por el autor.

\section{INSTRUMENTO}

De acuerdo con Maslach (1986), el manual del Malasch Burnout Inventory (MBI) mide la frecuencia y la intensidad con la que se sufre el BO. Las respuestas a las 22 preguntas miden tres dimensiones diferentes: agotamiento emocional (EE), despersonalización (D) y realización personal $(\mathrm{RP})$. 
- Agotamiento Emocional (EE): Su puntuación es directamente proporcional a la intensidad del síndrome; es decir, a mayor puntuación en esta subescala, mayor es el nivel de BO experimentado por el sujeto.

- Despersonalización (D): También su puntuación guarda proporción directa con la intensidad del BO.

- Realización Personal (RP): En esta dimensión, la puntuación es inversamente proporcional al grado de $\mathrm{BO}$; es decir, a menor puntuación de realización o logros personales más afectado está el sujeto.

Maslach (1986) argumenta que la relación entre puntuación y grado de $\mathrm{BO}$ es dimensional, es decir, no existe un punto de corte que indique si existe o no existe tal entidad. Por consiguiente, se distribuyeron los rangos de las puntuaciones totales de cada subescala en tres tramos que definen un nivel de BO experimentado como bajo, medio o alto. En la Tabla 1 se explican estos valores. También se presenta la distribución de las dimensiones, los ítems que abarca el instrumento, el total de ítems, así como la puntuación máxima para cada una de las tres dimensiones.

Tabla 1:

Distribución de las puntuaciones y dimensiones

\begin{tabular}{|c|c|c|c|}
\hline Dimensión & Ítems & $\begin{array}{c}\text { Total de } \\
\text { items }\end{array}$ & $\begin{array}{c}\text { Puntuación } \\
\text { máxima }\end{array}$ \\
\hline EE & $1,2,3,6,8,13,14,16$ y 20. & 9 & 54 \\
\hline D & $5,10,11,15$ y 22. & 5 & 30 \\
\hline RP & $4,7,9,12,17,18,19$ y 21 & 8 & 48 \\
\hline
\end{tabular}

Fuente: elaboración propia con base en los datos de Malasch (1986).

La Tabla 2 muestra los valores límites por cada nivel de incidencia del BO. 
Tabla 2:

Valores límites del BO

\begin{tabular}{|c|c|c|c|}
\hline Dimensión & $\begin{array}{c}\text { Nivel } \\
\text { Bajo }\end{array}$ & $\begin{array}{c}\text { Nivel } \\
\text { Medio }\end{array}$ & $\begin{array}{c}\text { Nivel } \\
\text { Alto }\end{array}$ \\
\hline EE & $\begin{array}{c}\text { Menor a } \\
18\end{array}$ & $\begin{array}{c}\text { Entre 19 } \\
\text { y 26 }\end{array}$ & Mayor a 27 \\
\hline D & $\begin{array}{c}\text { Menor } \\
\text { a 5 }\end{array}$ & Entre 6 y 9 & Mayor a 10 \\
\hline RP & $\begin{array}{c}\text { Mayor a } \\
40\end{array}$ & $\begin{array}{c}\text { Entre 34 } \\
\text { y 39 }\end{array}$ & $\begin{array}{c}\text { Menor a } \\
33\end{array}$ \\
\hline
\end{tabular}

Fuente: elaboración propia con base en los datos de Malasch (1986).

\section{AnÁLISIS DE LOS DATOS}

Se aplicó el cuestionario Maslach Burnout Inventory (1986). Los resultados proporcionaron medidas acerca de las tres dimensiones que conforman el instrumento: agotamiento emocional (EE), despersonalización (D) y realización personal (RP). Al interpretar estos valores, partimos del supuesto de que un ejecutivo afectado por este síndrome reportará valores altos de agotamiento emocional y de despersonalización, y en contraste, tendrá valores bajos de realización personal, pues este último indicador es una medida inversa a la relación de las dos primeras variables. La Tabla 3 muestra los resultados de las encuestas. En la recopilación se utilizaron colores distintivos para cada rubro perteneciente al BO.

Tabla 3:

Distribución de las puntuaciones y dimensiones

\begin{tabular}{|c|c|c|c|c|c|c|}
\hline Número & $\begin{array}{c}\text { Puntaje } \\
\text { EE }^{\mathbf{1}}\end{array}$ & Nivel & Puntaje $\mathbf{D}^{\mathbf{2}}$ & Nivel & Puntaje RP & Nivel \\
\hline 1 & 18 & Bajo & 6 & Medio & 24 & Alto \\
\hline 2 & 0 & Bajo & 7 & Medio & 36 & Medio \\
\hline 3 & 16 & Bajo & 0 & Bajo & 38 & Medio \\
\hline 4 & 0 & Bajo & 2 & Bajo & 43 & Bajo \\
\hline 5 & 38 & Alto & 16 & Alto & 31 & Bajo \\
\hline 6 & 5 & Bajo & 11 & Alto & 21 & Alto \\
\hline
\end{tabular}

${ }^{1}$ Agotamiento Emocional (EE).

2 Despersonalización (D).

${ }^{3}$ Realización personal (RP). 
Omar Alejandro Pérez Cruz

\begin{tabular}{|c|c|c|c|c|c|c|}
\hline Número & $\begin{array}{c}\text { Puntaje } \\
\text { EE }^{1}\end{array}$ & Nivel & Puntaje $D^{2}$ & Nivel & Puntaje RP $^{3}$ & Nivel \\
\hline 7 & 18 & Bajo & 2 & Bajo & 33 & Bajo \\
\hline 8 & 21 & Medio & 13 & Alto & 34 & Medio \\
\hline 9 & 16 & Bajo & 12 & Alto & 41 & Bajo \\
\hline 10 & 19 & Medio & 14 & Alto & 41 & Bajo \\
\hline 11 & 5 & Bajo & 6 & Medio & 42 & Bajo \\
\hline 12 & 10 & Bajo & 8 & Medio & 42 & Bajo \\
\hline 13 & 10 & Bajo & 4 & Bajo & 43 & Bajo \\
\hline 14 & 31 & Alto & 15 & Alto & 27 & Alto \\
\hline 15 & 18 & Bajo & 5 & Bajo & 40 & Bajo \\
\hline 16 & 11 & Bajo & 2 & Bajo & 34 & Medio \\
\hline 17 & 10 & Bajo & 2 & Bajo & 35 & Medio \\
\hline 18 & 28 & Alto & 16 & Alto & 26 & Alto \\
\hline 19 & 19 & Medio & 0 & Bajo & 29 & Alto \\
\hline 20 & 12 & Bajo & 2 & Bajo & 34 & Medio \\
\hline 21 & 33 & Alto & 19 & Alto & 20 & Alto \\
\hline 22 & 19 & Medio & 1 & Bajo & 34 & Medio \\
\hline 23 & 11 & Bajo & 8 & Medio & 40 & Alto \\
\hline 24 & 19 & Medio & 8 & Medio & 43 & Bajo \\
\hline 25 & 2 & Bajo & 0 & Bajo & 40 & Bajo \\
\hline 26 & 16 & Bajo & 5 & Bajo & 38 & Medio \\
\hline 27 & 1 & Bajo & 0 & Bajo & 48 & Bajo \\
\hline 28 & 11 & Bajo & 5 & Bajo & 40 & Bajo \\
\hline 29 & 3 & Bajo & 1 & Bajo & 48 & Bajo \\
\hline
\end{tabular}

Fuente: elaboración propia con base en los datos obtenidos. 


\section{Prueba de confiabilidad}

Una vez obtenidos los datos, realizamos una prueba de confiabilidad bifactorial, mediante la prueba de alfa de Cronbach. La prueba se aplicó a los resultados de las variables EE y RP, obteniendo un resultado de 0.7256 , lo cual es una calificación muy buena, lo que sugiere que ambas variables miden el mismo constructo de $\mathrm{BO}$.

\section{NIVELES DE BO}

Tomando los valores absolutos de alta incidencia para situar a un ejecutivo en el tramo alto de la escala, obtuvimos la siguiente distribución: el 13\% de la muestra presentó puntuaciones altas de EE y un $28 \%$ presentó puntuaciones altas en cuanto a D. En el caso de RP, un $49 \%$ obtuvo puntuaciones bajas ${ }^{4}$. Es importante notar que un $20 \%$ (6) de los ejecutivos presentaron puntuaciones "negativas" en dos o más de las dimensiones y un 3\% (1) en todos. La Tabla 4 muestra estos valores y porcentajes.

Tabla 4:

Valores de tendencia central y porcentajes de distribución

\begin{tabular}{|l|c|c|c|c|c|c|c|}
\hline & $\begin{array}{c}\text { Valores } \\
\text { resultantes }\end{array}$ & $\begin{array}{c}\text { Fre- } \\
\text { cuencia }\end{array}$ & $\begin{array}{c}\text { Valores } \\
\text { Bajos }\end{array}$ & $\begin{array}{c}\text { Fre- } \\
\text { cuencia }\end{array}$ & $\begin{array}{c}\text { Valores } \\
\text { Medios }\end{array}$ & $\begin{array}{c}\text { Fre- } \\
\text { cuencia }\end{array}$ & $\begin{array}{c}\text { Valores } \\
\text { Altos }\end{array}$ \\
\hline Media & & & & & & \\
\hline $\begin{array}{l}\text { Cansancio } \\
\text { emocional (EE) }\end{array}$ & 14,41 & 20 & $\mathbf{6 4 . 5 \%}$ & 5 & $\mathbf{1 6 . 1 \%}$ & 4 & $\mathbf{1 2 . 9 \%}$ \\
\hline $\begin{array}{l}\text { Despersonal- } \\
\text { ización (D) }\end{array}$ & 6,55 & 15 & $\mathbf{5 1 \%}$ & 6 & $\mathbf{2 1 \%}$ & 8 & $\mathbf{2 8 \%}$ \\
\hline $\begin{array}{l}\text { Realización } \\
\text { personal (RP) }\end{array}$ & 36,03 & 14 & $\mathbf{4 9 \%}$ & 8 & $\mathbf{2 7 \%}$ & 7 & $\mathbf{2 4 \%}$ \\
\hline
\end{tabular}

${ }^{4}$ Recordemos que esta dimensión es un indicador inverso entre más bajo existe una significancia negativa, a diferencia de las dos primeras dimensiones que la significancia negatividad se presenta a mayor puntuación. 


\section{Varianza estadística de BO}

Las desviaciones típicas de las medias encontradas para los tres constructos no presentan una varianza estadísticamente amplia, mayor de 10 puntos, lo que indica que en términos generales la dimensiones del BO se presentaron de manera muy homogénea. Se puede decir que existe una correlación entre las tres dimensiones, de forma que quienes presentaron niveles altos de EE también presentaron altos niveles de $\mathrm{D}$ y que estos altos niveles de EE y $\mathrm{D}$, se relacionan con bajos niveles de RP.

La Tabla 5 muestra una correlación positiva entre los niveles de RP y cualquiera de las otras dos variables. Este hallazgo es muy interesante, en la medida que sugiere que el EE y la D están provocados, al menos en buena parte, por aspectos de la baja realización personal. Este es un dato significativo, tomando en cuenta que solo un $3 \%$ de los participantes, presenta esta relación negativa en sus tres dimensiones.

Tabla 5:

Correlaciones

\begin{tabular}{|ll|r|r|r|}
\hline & & EE & D & \multicolumn{1}{c|}{ RP } \\
\hline EE & Correlación de Pearson & 1 & $.654(* *)$ & $-.540(* *)$ \\
& Sig. (bilateral) & & .000 & .003 \\
& $\mathrm{~N}$ & 29 & 29 & 29 \\
$\mathrm{D}$ & Correlación de Pearson & $.654(* *)$ & 1 & $-.449(*)$ \\
& Sig. (bilateral) & .000 & & .014 \\
& $\mathrm{~N}$ & 29 & 29 & 29 \\
$\mathrm{RP} \quad$ Correlación de Pearson & $-.540(* *)$ & $-.449(*)$ & 1 \\
& Sig. (bilateral) & .003 & .014 & \\
$\mathrm{~N}$ & 29 & 29 & 29 \\
\hline
\end{tabular}

** La correlación es significativa al nivel 0,01 (bilateral).

* La correlación es significante al nivel 0,05 (bilateral).

Fuente: resultados analizados en SPSS. 
En la Tabla 6 se resumen los valores resultantes y los valores límites.

Tabla 6:

Valores resultantes y límites del BO

\begin{tabular}{|l|c|c|c|c|}
\hline & \multicolumn{2}{|c|}{$\begin{array}{c}\text { Valores } \\
\text { resultantes }\end{array}$} & $\begin{array}{c}\text { Valores } \\
\text { límites }\end{array}$ & $\begin{array}{c}\text { Varianza } \\
\text { del límite }\end{array}$ \\
\hline & Media & $\begin{array}{c}\text { Desv. } \\
\text { típ. }\end{array}$ & Límite & \\
\hline Cansancio emocional (EE) & 14,41 & 9,77 & $>27$ & $\begin{array}{c}53 \% \text { por debajo } \\
\text { del límite superior }\end{array}$ \\
\hline Despersonalización (D) & 6,55 & 5,69 & $>10$ & $\begin{array}{c}45 \% \text { por debajo } \\
\text { del límite superior }\end{array}$ \\
\hline Realización personal (RP) & 36,03 & 7,35 & $<33$ & $\begin{array}{c}\text { 9\% por encima del } \\
\text { límite superior }\end{array}$ \\
\hline
\end{tabular}

Fuente: elaboración propia con base en los resultados y los datos de Malasch (1986).

También, tomamos en cuenta los valores relativos. Además de analizar las dimensiones negativas, dirigimos la mirada a aquellos ejecutivos que están en riesgo de padecer el síndrome. Para ello, identificamos los valores que se encuentran en los niveles medioalto para las dimensiones de EE y D, y medio-bajo para RP. De esta forma, obtuvimos los siguientes resultados. Con respecto al EE, un $36 \%$ (9 incidencias) de la muestra presenta valores altos-medio. Un $51 \%$ (15 incidencias) presenta valores altos-medio de D y un $69 \%$ (20 incidencias) presenta un valor bajo-medio RP. Estos indicadores demuestran que un $52 \%^{5}$ del personal está en riesgo de adquirir el síndrome. Si relacionamos estos resultados con el hecho de que el porcentaje más alto de puntuaciones negativas (48\%) se observa en la dimensión RP, podemos concluir que el síndrome de BO se debe a la falta de oportunidades de desarrollo entre los ejecutivos del centro y por una falta de apego al grupo de trabajo, lo que resulta en una carencia de identidad organizacional. Podemos también decir que esta baja en la realización personal, se puede deber a un estilo

\footnotetext{
${ }^{5}$ Este porcentaje se obtuvo al obtener la media entre los 3 porcentajes anteriores.
} 
de liderazgo orientado a las tareas y a los resultados, tal como lo señaló Davis (2004). Este autor sostiene que este liderazgo orientado a las tareas suele generar ambientes de trabajo con características de apatía, falta de compromiso y desinterés por parte de los trabajadores, además del sentimiento de falta de apreciación por sus contribuciones y, a su vez, desgano por sus actividades laborales cotidianas.

\section{ComentARIOS SObRe LOS RESUltados Del ESTUdiO}

Este estudio es la primera investigación que evalúa el $\mathrm{BO}$ en los ejecutivos del centro de emergencias de Colima. Por lo tanto, no podemos establecer comparaciones referenciadas.

Como resultados importantes, se pudo apreciar que, pese a que los ejecutivos del centro de emergencias de Colima, la presencia del síndrome de $\mathrm{BO}$ se presentó solo en un 3\% en la muestra. Sin embargo, es destacable que un $52 \%$ del personal está en riesgo de adquirir el síndrome.

Otro de los hallazgos destacables fue la existencia de una correlación positiva entre las tres dimensiones evaluadas. Esto demuestra una consistencia interna del instrumento y su utilidad para continuar aplicándolo.

Es importante mencionar que en esta investigación se seleccionó una unidad de análisis que no había sido objeto de estudio hasta el momento, esto es, el personal de emergencias 066, el que debido a que está en contacto telefónico con otras personas, no recibe el mismo nivel de tensión que otros telefonistas. Es por ello que se espera que más investigadores se interesen en estudiar este fenómeno.

Una de las limitaciones de este estudio es su carácter transversal (en tiempo presente). La investigación puede servir como punto de partida para un estudio que comprenda varias etapas en la vida laboral de los ejecutivos y del personal del centro de emergencias de Colima.

Para estudios futuros se recomienda complementar la aplicación del instrumento con variables como género, estado civil, antigüedad en la empresa, entre otras. Esto ayudaría a determinar los efectos de $\mathrm{BO}$ en diferentes poblaciones, como por ejemplo las mujeres $\mathrm{u}$ hom- 
bres con familia. Tomando como base los resultados aquí obtenidos, creemos que es imprescindible realizar un plan de intervención en este centro de emergencias. El plan debe considerar técnicas de autocontrol y manejo del estrés que influyan en el optimismo y la autoestima. 


\section{Referencias}

Bernaldo de Quirós-Aragón, M. y Labrador-Encinas, F. (2007). Evaluación del estrés laboral y burnout en los servicios de urgencia extrahospitalariaria. International Journal of Clinical and Health Psychology, 7(2), 323-335.

Benevides Pereira, A., Justo, T., Batista Gomes, F., Martins Silva, S. y Volpato, D. (2003, enero-diciembre). Sintomas de estresse em educadores brasileiros. Aletheia, 17-18, 63-72.

Cano, A. (2012). La naturaleza del estrés. Sociedad española para el estudio de la ansiedad y el estrés. Disponible en: http://www.ucm.es/info/seas/estres_lab/el_estres.htm

Davis, K. N. (2004). Human behavior at work. New York, Estados Unidos: Mc Graw Hill.

Dennis, C. (2004). Psicología. D.F., México: Thomson Editores S.A de C.V.

Disegni, C. (2002). Madres trabajadoras sufren hasta 3 veces mayores niveles de estrés. Madrid, España: La Tercera.

Escobar, A. y Gomez, B. (2006). Estrés y memoria. Revista Mex Neuroci, 7(1):8-14.

Fernández, P. (2010, noviembre). La influencia de los factores estresantes del trabajo en el rendimiento laboral. Invenio, 13(25), 111-124.

Guerrero, J. (2003). Los roles no laborales y el estrés en el trabajo. Revista Colombiana de Psicología, 012, 73-84.

Gil-Monte, P. R. (2005). El sindrome de quemarse en el trabajo (burnout). Una enfermedad laboral en la sociedad del bienestar. Madrid, España: Ediciones Pirámide.

Gil-Monte, P. R. y Peiro, J. (1999). A study on significant sources of the "burnout syndrome" in workers at occupational centers for the mentally disabled. Psychology in Spain, 2(1), 116-123.

Guillén, G. G. (2000). Psicología del trabajo para las relaciones laborales. D.F., México: McGraw Hill.

Lazarus, R. (1984). Stress appraisal and coping. New York, Estados Unidos: Editorial Springer.

Lazarus, R. y Folkman, S. (1991). Estrés y procesos cognitivos. D.F., México: Ed. Roca. 
Maslach, C. Y. (1986). The Maslach Burnout Inventory. Manual (2nd ed.). Palo Alto, CA.: Consulting Psychologists Press.

Moreno, B., Oliver, C., Pastor, J. y Aragoneses, A. (1990). El burnout, una forma específica de estrés laboral. En V. E. Carballo y G. Buela. (Comp.), Manual de psicología clínica. Madrid: Siglo XXI.

Peiró, J. M. (2005). Desencadenante del estrés laboral. Madrid, España: Ediciones Pirámide.

Peiró, P. R. (1999). Perspectivas teóricas y modelos interpretativos para el estudio del síndrome de quemarse por el trabajo. Anales de Psicología, 15(002), 261-268.

Peralta, M. (2006, julio). Manifestaciones del acoso laboral, mobbing y síntomas asociados al estrés postraumático: estudio de caso. Psicología desde el Caribe, 117, 1-26.

Phyllis, G. M. (2000). Mental health in the workplace. Introduction. Ginebra: Organización Internacional del Trabajo.

Robbins, S. (2003). Organizacional behavior. New York, Estados Unidos: Pearson Educación.

Salgado, M. (17 de abril de 2007). Los daños del estrés en exceso. Periódico El Economista. D.F., México.

Schaufeli, W., Leiter. M. y Maslach, C. (2009). Burnout: 35 years of research and practice. Career Development International, 14(3), 204-220. 